\title{
Engineering calculations for complex geometric domains
}

\author{
Elzbieta Gawronska ${ }^{1, *}$, Robert Dyja ${ }^{1}$, Andrzej Grosser ${ }^{1}$, Jerzy Winczek ${ }^{1}$ \\ ${ }^{1}$ Faculty of Mechanical Engineering and Computer Science, Czestochowa University of Technology, \\ Dabrowskiego 69, 42-201 Czestochowa, Poland
}

\begin{abstract}
The paper aims to present the possibility of performing engineering calculations in domains of complex shapes expressed by mathematical descriptions. We focus on calculations done with the use of the Finite Element Method. An example of the compound structure is gyroid, which is a periodic structure representing an area close to the porous structure. The presented gyroid structure exhibits circular struts and a spherical core and spatially occupies a cube. The side length of the cube is defined as unit cell size, and the volume percentage of the struts inside the cube is referred to as volume fraction. The periodic cellular lattice structures are generated by our own software module. One of the uses of such structures is their employment in additive manufacturing (AM) of the so-called 3D printing (layer-by-layer AM technique), where they can contribute reducing the weight of an item and limiting the material consumption. It is important to answer the question of what effect does an element with a gyroid structure have on the thermal properties (e.g. the heat flow versus the volume fraction) compared to an element with full structure. The paper will show the results of such a comparison.
\end{abstract}

Keywords: gyroid, volume fraction, complex shapes, heat flow, numerical modeling

\section{Introduction}

$3 \mathrm{D}$ printing was invented in 20th century, but its industrial use was quite limited. It has changed over the last years. Additive Manufacturing (AM) processes take information from a computer-aided design (CAD) file that is later converted to a stereolithography (STL) file. $\mathrm{CAD}$ systems are dedicated to the design of parts manufactured by traditional methods and are not helpful to designers who want to exploit the extensive opportunities offered by AM technology. For this process, the drawing made in CAD software is approximated by discrete elements and sliced to contain the information of each layer that is going to be printed. AM technologies build near-net shape components layer by layer using 3D model data and so they are the direct descendants of the work in 3D printing are revolutionary to many sectors of manufacturing insofar as they reduce part lead time, cost, material waste, and energy usage [1]. Furthermore, AM has the potential to enable modern designing that could not be manufactured using conventional methods and to extend the life of in-service parts through innovative repair methodologies. Applications, where legacy components are

\footnotetext{
* Corresponding author: gawronska@icis.pcz.pl

Reviewers: Vladimir Dekýš, Ivan Kuric
} 
still necessary for operation and fabricators, no longer manufactured, for instance aging aircraft systems or older power stations, could use AM to create parts directly from a CAD file. For industrial sectors, one of the most crucial values of additive manufacturing is the ability to reduce weight while maintaining mechanical performance. The advantages are conclusive since AM can result in lower material costs, significant reductions in production time and increasing design flexibility [2]. AM is transforming approaches in medicine and making work easier for architects. For consumer products, artistic and aesthetic designs can be directly manufactured from a CAD file without concern for standard manufacturing practice. Many of the traditional designs for traditional production principles can no longer be applicable if parts with complex internal features have to be produced. AM technology is used to create elements previously difficult or impossible to be manufactured with traditional techniques [3].

One of the main advantages of AM methods is the ability to produce parts which have very complex geometry and which are made by prevailing methods in a very complicated way. For AM technology to be included in the industrial production of the real components, that high mechanical properties of produced parts are achieve. Moreover, a geometrically defined structure with complex shapes which allows weight reduction must have sufficient toughness. The final properties of manufactured parts are strongly dependent on each laser produced track and layer [4].

Gyroid structure is a complex structure which allows weight reduction and has circular and smooth struts with a spherical core. The circular and smooth struts of the unit cell continuously varying along the spherical core are presented in Fig. 1.

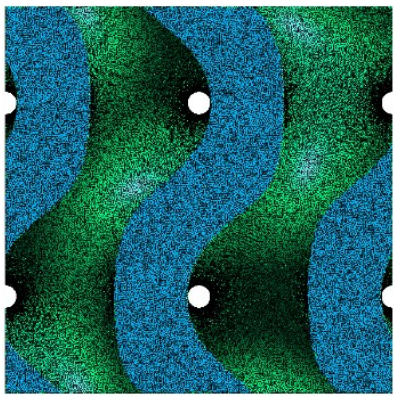

a)

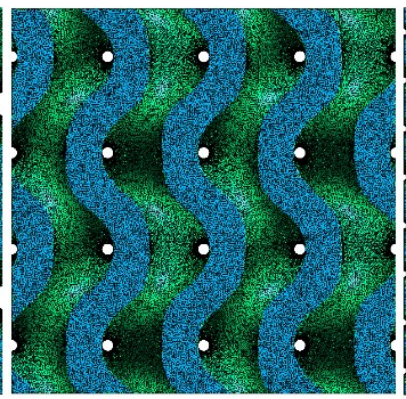

b)

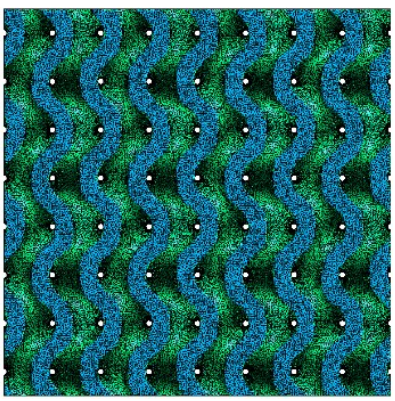

c)

Fig. 1. View through gyroid structures with a) one b) two c) four unit cells per one dimension

Additionally, the design of gyroid structures allows easy removal of the loose metal powder trapped inside of these structures after the building process of a particular part is completed. This inter-connected network of an open structure is well suited for unnecessary material removal [5]. The concept of designed cellular lattice materials is motivated by the desire to put material only where it is needed for a particular application. From a mechanical engineering viewpoint, a key advantage offered by cellular materials is high strength accompanied by a relatively low mass. These materials can provide excellent energy absorption characteristics as well good thermal and acoustic insulation properties. Designed cellular structures typically exhibit stronger structure strength per unit weight than typical foam structures [6].

In the paper, we use gyroid structure which is a homogeneous, isotropic structure, preserving the same mechanical properties in each direction. A team of researchers at MIT has designed one of the strongest lightweight materials known and has made the simulations with 3-D gyroid forms created from this matter. The results of tensile and compression tests showed that the construction could be ten times as strong as steel but 
much lighter. Their findings proved that the crucial aspect of performed tests has more to do with the unusual geometrical configuration than with the material itself. The researchers suggest that similar durable, lightweight materials could be made from a variety of matter by creating similar geometric features [7]. Isotropy of that structures is very useful because of there is no difference in the physical properties such as thermal expansion, heat conduction, electrical conductivity, refractive index, etc. regardless of the direction in which they are measured. In the paper, we consider heat flow. Results revealed that the type of structure and volume fraction are the main factors influencing the heat flow pace.

\section{Methods}

When dealing with solid 3D objects, it is often convenient to only model the object's boundaries using a mathematical representation of the surfaces. The choice of surface representation is particularly important for the computational modeling of $3 \mathrm{D}$ objects as each depiction has its advantages and disadvantages. These include the availability and complexity of operations that can be used to manipulate the surface (e.g. smoothing, Boolean operations) as well as the efficiency of the representation. Each formula also has an impact on how models are visualized and ultimately realized (e.g. via AM). The most common surface's depiction can be classified as one of the following forms: explicit, parametric or implicit.

The most common type of explicit surface representation is a mesh of polygons. Typically, these are triangles or quadrilaterals although others can be used. These polygons are often stored as an order list of vertex indices - the order being used to define the direction the polygon is facing (i.e., the surface normal) [8].

Unlike explicit surfaces, parametric surfaces do not store points on a surface. Instead, points on a parametric surface are expressed as a function of the parametric variables $(\mathrm{u}, \mathrm{v})$, which can be generalized to lie on the unit square $[0,1] \times[0,1]$. Non-Uniform Rational BSpline (NURBS) surfaces are a form of parametric surface commonly used due to their compact representation, smooth surfaces and easy of manipulation $[9,10]$.

Implicit surfaces are defined as an iso-surface of some function $\mathrm{f}$. In $3 \mathrm{D}$ the surface is defined by a set of points $\mathrm{p} \in \mathrm{R} 3$ satisfying the equality:

$$
f(x, y, z)=0 .
$$

As with parametric forms, implicit surfaces provide a compact representation for potentially complex surfaces. They also offer some advantages, notably their flexibility and well-defined Boolean operations. However, unlike parametric forms they provide little local shape control and manipulating them can be unintuitive. The implicit function approach for the structure design being used has been proved to be very versatile, as it allows geometries to be conceived just by pure mathematical expressions. This way, different cellular structures can be easily defined and optimized [11]. In the paper, gyroid lattice structures are mathematically defined, allowing precise control of volume fraction (depends on $\delta$ thickness) and unit cell size in the creation of an interconnected network:

$$
\cos x \sin y+\cos y \sin z+\cos z \sin x=\delta,
$$

thickness is normalized and takes values from 0 to 1 . In the paper, it takes value $\delta=0.8$.

The design of gyroid structures is based on efficiently generated and implicitly defined periodic structure and rapidly constructed finite element meshes. Finite element mesh for the full cube was generated with Gmsh software. The used version of Gmsh did not have the option of design NURBS surface, mesh for gyroid structure was generated with our own 
software using CGAL library (it contains procedures for describing the division of an area into finite elements). CGAL is a software project that provides easy access to efficient and reliable geometric algorithms in the form of a $\mathrm{C}++$ library. CGAL is used in various areas which need geometric computation, such as geographic information systems, computer aided design, molecular biology, medical imaging, computer graphics, and robotics [12].

Gyroid lattice structures are mathematically defined, allowing precise control of volume fraction and unit cell size in the creation of an interconnected network. The volume fraction is defined as the volume percentage of the solid material in the cellular lattice structure. The gyroid surface was used to generate periodic lattice structures with the same unit cell sizes $u=0.4[\mathrm{~m}]$ and average volume fraction of $\gamma \approx 0.52$. The designed models of the gyroid with the finite element meshes (built in the cube presented in Fig. 2) generated for every considered lattice structure are shown in Fig. 3, Fig. 4 and Fig. 5.

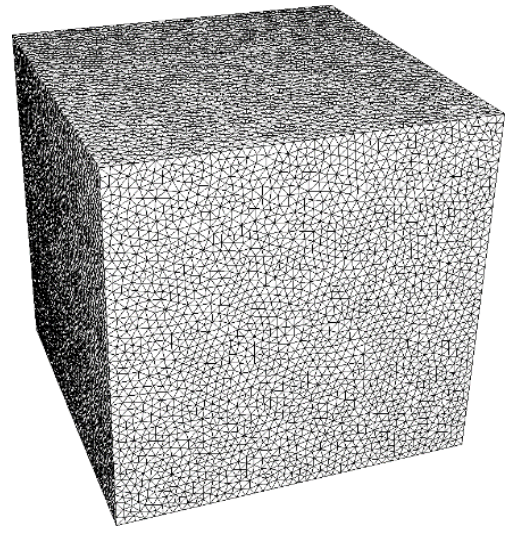

Fig. 2. Cube mesh

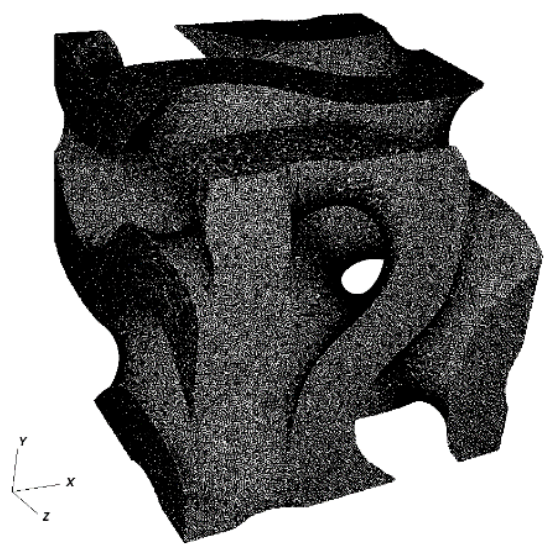

Fig. 3. Gyroid mesh (one unit cell per one dimension)

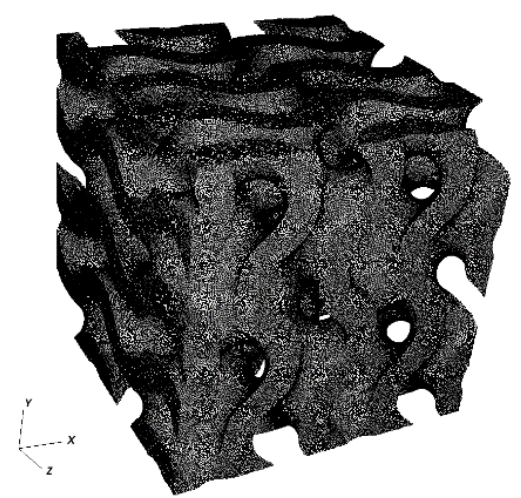

Fig. 4. Gyroid mesh (two unit cells per one dimension)

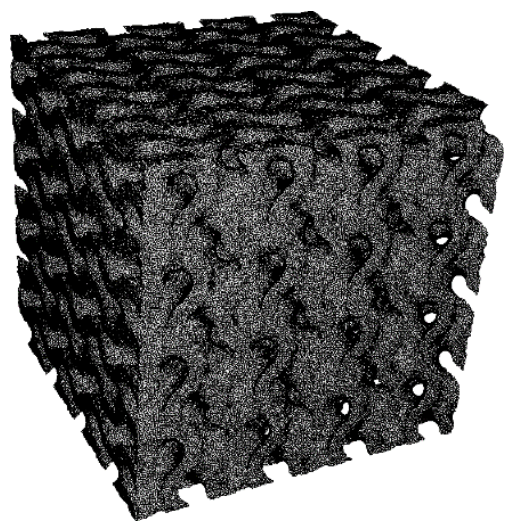

Fig. 5. Gyroid mesh (four unit cells per one dimension) 


\section{Numerical simulations}

The article presents the results of the heat conduction simulations for a cube whose face has $0.04[\mathrm{~m}]$ edge length and for the gyroid lattice structures inserted in the cube. The following physical properties were used in simulations: $\lambda$ - heat transfer coefficient $260[\mathrm{~W} /(\mathrm{m} \cdot \mathrm{K})], \rho$ - density $2800\left[\mathrm{~kg} / \mathrm{m}^{3}\right], c$-specific heat $1000[\mathrm{~J} /(\mathrm{kg} \cdot \mathrm{K})]$. Such material properties are similar to those of aluminum alloys - material significant and often used in industry. The boundary condition is a heat flux established on one surface with a fixed value of $10000\left[\mathrm{~W} / \mathrm{m}^{2}\right]$ (Neumann boundary condition). Four simulations were made: one for the full cube and three for the three different areas of the gyroid. For each simulation, 500-time steps of 0.5 [s] were made. The initial temperature is 300 [K]. The temperature distributions and their values are shown in Fig. 6, Fig. 7, Fig. 8 and Fig. 9 for the cube, the gyroid with one unit cell per dimension (gyroid-1), the gyroid with two unit cells per dimension (gyroid-2) and the gyroid with four unit cells per dimension (gyroid-4), respectively.

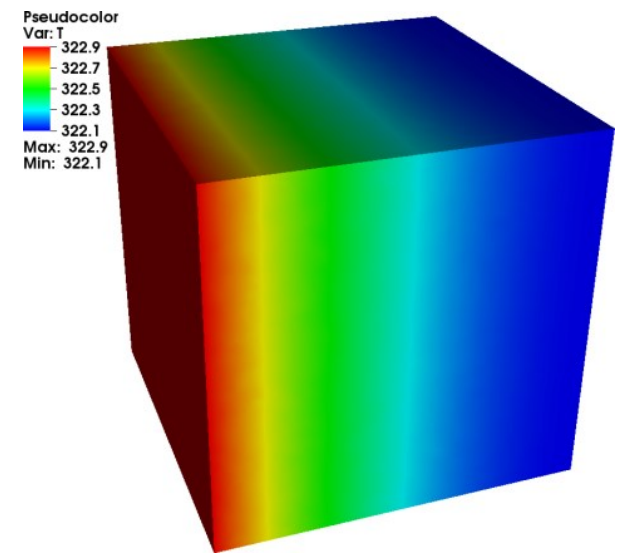

Fig. 6. The temperature distribution after 500 time steps - cube

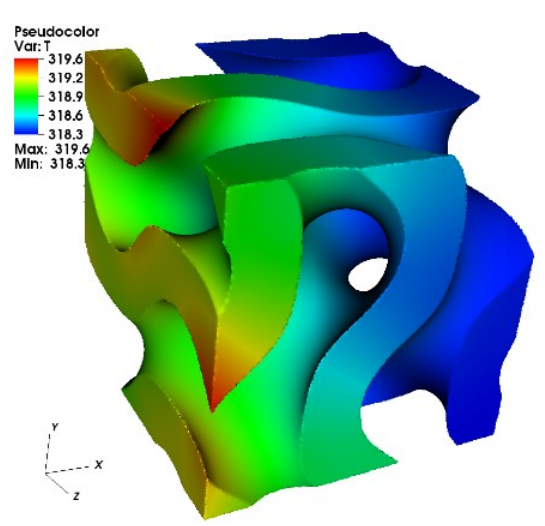

Fig. 7. The temperature distribution after 500 time steps - gyroid with one unit cell per one dimension

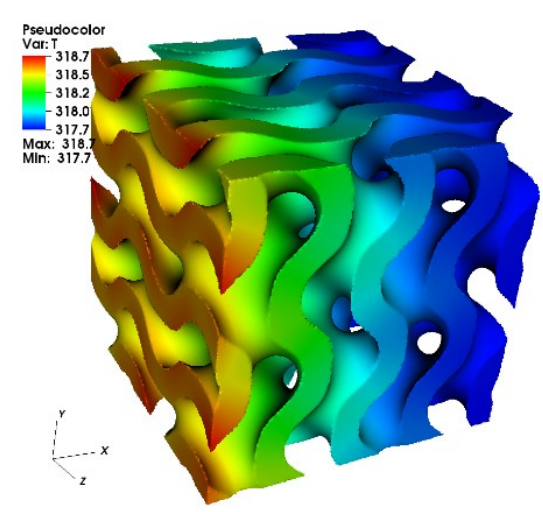

Fig. 8. The temperature distribution after 500 time steps - gyroid with two unit cells per one dimension

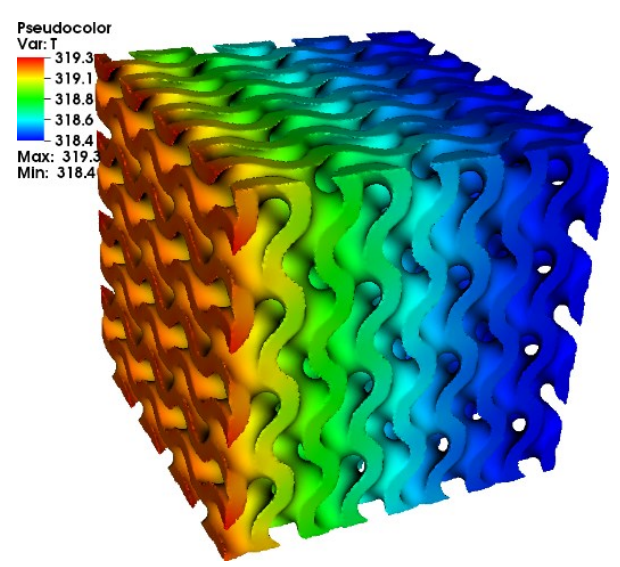

Fig. 9. The temperature distribution after 500 time steps - gyroid with four unit cells per one dimension 
The gyroid meshes have much more finite elements than the cube since it was necessary to use ones which are finer to accurately recreate all details of the geometry (see Table 1).

Table 1. Characteristics of domain decomposition

\begin{tabular}{|c|c|c|}
\hline Structure & Number of nodes & Number of elements \\
\hline Cube & 44567 & 241939 \\
\hline Gyroid-1 & 289543 & 1195995 \\
\hline Gyroid-2 & 469847 & 1823180 \\
\hline Gyroid-4 & 814535 & 3044948 \\
\hline
\end{tabular}

Table 2 summarizes the data refers to the time that was needed to achieve the minimum temperature of $315[\mathrm{~K}]$ (differences in calculated temperatures do not exceed $0.01[\mathrm{~K}]$ ). Of course, this temperature was at the furthest possible distance from the wall on which the boundary condition was set. The boundary condition was set only on one wall. The rest have nothing to set, which in case of heat conduction means excellent insulation. There is no air inside the gyroid structure or any heat exchange, just heat flows from the heated wall through the entire volume of the structure to the opposite wall.

Table 2. Characteristics of values important in numerical simulations

\begin{tabular}{|c|c|c|c|}
\hline Structure & $\begin{array}{c}\text { Area } \\
\text { fraction } \\
\boldsymbol{\varepsilon}\end{array}$ & $\begin{array}{c}\text { Volume } \\
\text { fraction } \\
\boldsymbol{\gamma}\end{array}$ & $\mathbf{t}[\mathbf{s}]$ \\
\hline Cube & 1 & 1 & 170.0 \\
\hline Gyroid-1 & 0.4336769 & 0.5215625 & 206.0 \\
\hline Gyroid-2 & 0.4203725 & 0.5223438 & 212.5 \\
\hline Gyroid-4 & 0.4265122 & 0.5228375 & 205.0 \\
\hline
\end{tabular}

The presented data showcase that the gyroid structures heat up in almost the same time. That time, however, is longer than the full cube heating time. This difference can be explained by the fact that for an established boundary condition, the heat flux acting on the surface unit depends on a surface occupied by, e.g. a gyroid on the selected wall. By defining the area fraction $\varepsilon$ as the ratio of the area held by a chosen structure $a^{\prime}$ to the area of the entire wall $a$ :

$$
\varepsilon=\frac{a^{\prime}}{a}
$$

for the cube a maximum value $\varepsilon_{\max }=1$ is obtained, while it is the smallest for the gyroid-2 structure. It appears that the amount of heat that flowed into the cube was the largest and thus the process of heating up was the fastest there, whereas for the structure of gyroid-2 the amount of heat was the smallest, so the structure was heating up the slowest. Using this property of gyroidal structures can be substantial for the industry in situations in which a given product should not get heated too quickly during its use.

\section{Conclusions}

The research conducted in this paper concerned engineering calculations for complex geometric domains. Gyroid structures are a good example of such domains. The use of mathematical representations in an implicit formulation has been presented to be quite 
straightforward and flexible for the generation of gyroid lattice structures. To recreate the gyroidal structure, this is needed much more of finer finite elements. There were conducted several numerical simulations with authorial software, and it was shown the achieved results of the heat flow through the complex shape of a structure are physically correct. The influence of area and volume fractions on heating up of chosen structure were settled - the gyroidal structures accumulate heat slower than full cubes, which can be substantial for the industry in situations in which a given product should not get heated too quickly during its use.

In future work, the realization of research for the gyroid structure with different geometry, various area and volume fractions, and different wall thickness is planned. Rapid heating of materials preceding rapid solidification induces high thermal variations resulting in residual stresses that can introduce cracks or undesired effects to the machine part. It is also an important aspect of future considerations.

\section{References}

1. H. Piili, A. Happonen, T. Väistö, V. Venkataramanan, J. Partanen, A. Salminen, Cost Estimation of Laser Additive Manufacturing of Stainless Steel. $15^{\text {th }}$ Nordic Laser Materials Processing Conference, Nolamp, Physics Procedia 78, 388-396 (2015)

2. C. Yan, L. Hao, A. Hussein, D. Raymont, Evaluations of cellular lattice structures manufactured using selective laser melting. Int. J. Mach. Tool Manu. 62, 32-38 (2012)

3. K. V. Wong, A. Hernandez, A Review of Additive Manufacturing. ISRN Mechanical Engineering 2012, 208760, (2012)

4. K. Monkova, P. Monka, Some Aspects Influencing Production of Porous Structures with Complex Shapes of Cells. Proceedings of 5th International Conference on Advanced Manufacturing Engineering and Technologies: NEWTECH 2017, 267-276 (2017)

5. C. Beyer, D. Figueroa, Design and Analysis of Lattice Structures for Additive Manufacturing, J MANUF SCI E-T ASME 138 (12), 121014 (2016)

6. H. Liang, R. D. Y. Chunze, H. Ahmed, P. Young, Design and additive manufacturing of cellular lattice structures. The International Conference on Advanced Research in Virtual and Rapid Prototyping (VRAP), Leiria, Innovative Developments in Virtual and Physical Prototyping, 249-254 (2011)

7. D. L. Chandler, 3-d-graphene-strongest-lightest-materials. [Online] http://news.mit.edu/2017/3-d-graphene-strongest-lightest-materials-0106 (2017)

8. P. Zhang, J. Toman, Y. Yu, E. Biyikli, M. Kirca, M. Chmielus, A. C. To, Efficient Design-Optimization of Variable-Density Hexagonal Cellular Structure by Additive. Manufacturing: Theory and Validation, J. Manuf. Sci. E. 137 (2), 021004, 0104 (2015)

9. L. Yang, O. Harrysson, D. Cormier, H. West, H. Gong, B. Stucker, Additive Manufacturing of Metal Cellular Structures: Design and Fabrication. JoM 67 (3), 608-615 (2015)

10. D. Ma, F. Lin, C. Chua, Rapid Prototyping Applications in Medicine. Part 1: NURBSBased Volume Modelling, INT J ADV MANUF TECH 18 (2), 103-117 (2001)

11. G. Strano, L. Hao, R. M. Everson, K. E. Evans, A new approach to the design and optimisation of support structures in additive manufacturing. INT J ADV MANUF TECH 66 (9), 1247-1254 (2013)

12. https://www.cgal.org/ [Online] (Accessed 2308 2017) 